\title{
Qualidade de vida de idosos institucionalizados em cuidados paliativos
}

\author{
Quality of life of institutionalized elderly people in palliative care
}

Calidad de vida de ancianos institucionalizados en cuidados paliativos

Tereza Lais Menegucci Zutin ${ }^{1 *}$, Flavia Vilas Boas Ortiz Carli ${ }^{1}$, Thais Menegucci ${ }^{1}$, Mariana Cadelca Zalbinate $^{1}$, Anna Cláudia Dias Bossoni ${ }^{1}$, Kamila Cristina Viana ${ }^{1}$, Letícia dos Santos Oliveira ${ }^{1}$, Isabela Barrile Fernandes ${ }^{1}$, Pedro Henrique Menegucci Zutin².

\section{RESUMO}

Objetivo: conhecer e descrever a percepção de qualidade de vida dos pacientes internados em instituição de longa permanência sob o olhar do próprio doente. Métodos: Trata-se de um estudo de natureza observacional, exploratório-descritivo, transversal, realizado em uma Instituição de longa permanência para idosos do Interior Paulista, durante o período de Outubro a Dezembro 2018. A população do estudo foi composta por pacientes portadores de doença crônica ameaçadora da vida, entre outras patologias severas. Para a coleta de dados foi utilizado um formulário elaborado pelo pesquisador e também foi aplicada a Palliative Scale Outcames Scale (POS). Resultados: O número de internados na referida instituição totaliza 53 pacientes. Destes, 30 pacientes não apresentavam condições cognitivas para responder o formulário, portanto 23 idosos o fizeram. Obtivemos a prevalência do sexo feminino, cor branca, estado civil viúvo, idade superior a 70 anos, grau de instrução sem ciclo educacional completo, profissão trabalhador rural, religião católica, renda familiar de um salário mínimo, com presença de cuidador, portadores de doença de base. Conclusão: Conclui-se que a qualidade de vida desses pacientes em Instituições de longa permanência é satisfatória e atende às necessidades físicas, sociais e psicológicas diante de suas realidades e limitações apresentadas.

Palavras-chave: Qualidade de vida, Cuidados paliativos, Doente terminal.

\section{ABSTRACT}

Objective: know and describe the perception of quality of life of patients admitted to a long-term institution under the eyes of the patient. Methods: This is an observational, exploratory-descriptive, cross-sectional study, carried out in a long-term institution for the elderly in the interior of São Paulo, from October to December 2018. The study population consisted by patients with life-threatening chronic disease, among other severe pathologies. For data collection, a form prepared by the researcher was used and the Palliative Scale Outcames Scale (POS) was also applied. Results: The number of hospitalized in the referred institution totals 53 patients. Of these, 30 patients did not have cognitive conditions to answer the form, so 23 elderly people did so. We obtained the prevalence of females, white color, widowed marital status, age over 70 years, education level without complete educational cycle, profession, rural worker, Catholic religion, family income of one minimum wage, with presence of caregiver, patients with illness base. Conclusion: It is concluded that the quality of life of these patients in long-term institutions is satisfactory and meets the physical, social and psychological needs in view of their presented realities and limitations.

Key words: Quality of life, Palliative care, Terminally ill.

\footnotetext{
${ }^{1}$ Universidade de Marília - UNIMAR, Marília - SP. *E-mail: lais_zutin@hotmail.com

${ }^{2}$ Faculdade de Medicina de Marília - FAMEMA, Marília - SP.
}

SUBMETIDO EM: 12/2019

ACEITO EM: 1/2020

PUBLICADO EM: $3 / 2020$

REAS/EJCH | Vol.Sup.n.43 | e2790 | DOI: https://doi.org/10.25248/reas.e2790.2020 Página 1 de 9 


\section{RESUMEN}

Objetivo: conocer y describir la percepción de calidad de vida de estos pacientes hospitalizados en una institución a largo plazo bajo los ojos del propio paciente. Métodos: Este es un estudio observacional, exploratorio-descriptivo, transversal, celebrada en un centro de atención a largo plazo para ancianos en el interior de São Paulo, de octubre a diciembre de 2018. La población del estudio fue compuesta por pacientes con enfermedades crónicas potencialmente mortales, entre otras patologías graves. Para la recopilación de datos, se usó un formulario preparado por el investigador y también se aplicó la OutcamesScale (POS). Resultados: El número de hospitalizados en esta institución totaliza 53 pacientes. De estos, 30 pacientes no tenían condiciones cognitivas para responder el formulario, para tanto, 23 lo hicieron. Obtuvimos la prevalencia del estado civil femenino, blanco, viudo, edad mayor de 70 años, nivel educativo sin ciclo educativo completo, profesión trabajador rural, religión católica, ingreso familiar de un salario mínimo, con presencia de cuidador, personas con enfermedad de base. Conclusión: se concluye que la calidad de vida de estos pacientes en instituciones de atención a largo plazo es correspondiente y satisfactoria a sus necesidades físicas, sociales y psicológicas en vista de sus realidades y limitaciones.

Palabras clave: Calidad de vida, Cuidados paliativos, Enfermo terminal.

\section{INTRODUÇÃO}

O Cuidado Paliativo está associado ao termo "Hospice", fazendo referência aos abrigos destinados a receber e cuidar de viajantes e peregrinos no século V. Posteriormente, tornou-se uma instituição que abrigava órfãos, pobres e doentes, com características de hospitais. Já em 1967, Cicely Saunders fundou "St. Christopher's Hospice" um centro de assistência, ensino e pesquisa, o qual deu início ao estudo de controle da dor (SILVA MMP, 2014).

A Organização Mundial da Saúde definiu em 1990 "cuidados paliativos" propondo um cuidado ativo aos pacientes com a intenção de promover o alívio da dor e dos demais sintomas, afirmar a vida e considerar a morte um processo normal, não acelerar nem adiar, integrar os aspectos psicológicos e espirituais no cuidado ao paciente, oferecer um sistema de suporte que o possibilite viver tão ativamente quanto possível, até o momento da sua morte. Suporte para auxiliar os familiares durante a doença do ente querido e a enfrentar $o$ luto com abordagem. (ARAÚJO MMT e SILVA MJP, 2007).

Qualidade de vida é a percepção do indivíduo dentro de uma cultura e dos valores nos quais está inserido, além da relação com os objetivos, expectativas, padrões e preocupações. Na área da saúde, nasce nos séculos XVIII e XIX, a preocupação com a associação entre qualidade de vida e saúde. A qualidade de vida quando relacionada aos cuidados no final da vida apresenta desafios dentre os quais se destacam sociais, espirituais e psicológicos (BYOCK I, 1997). Pela perspectiva dos pacientes, qualidade de vida é receber adequado controle de dor e dos sintomas, evitando o prolongamento do sofrimento, e fortalecendo os relacionamentos com seus entes queridos.

Diante da realidade socioeconômica atual observa-se o envelhecimento populacional com aumento de doenças crônico-degenerativas. Nosso país colocou em vigência a Política Nacional do Idoso em 1964, onde se preza o cuidado familiar ao idoso em detrimento do atendimento asilar. Tal política influencia na qualidade de vida, já que de acordo com um estudo feito por Dalla Vecchia R, et al., (2005) 49\% dos idosos respondem que a vivência social e os vínculos familiares é o que simboliza a qualidade de vida e segundo Neri AL (2005) é no meio familiar em que há procura de segurança e apoio. Portanto, as relações familiares estão intimamente relacionadas com a qualidade de vida, trazendo benefícios aos idosos. Porém, Caldas CP (2002) destaca que mesmo sendo a família o foco principal no cuidado, muitas vezes é a falta de suporte que leva a institucionalização dos idosos. Por isso vê-se hoje um grande aumento das Instituições de Longa Permanência.

Avaliar a qualidade de vida em pacientes em cuidados paliativos é essencial para formação de sua condição e avaliação do serviço oferecido. Dessa forma, utiliza-se a Palliative Outcames Scale (POS), uma 
escala simples que se avalia a qualidade de vida e monitora o número e a intensidade com que os sintomas ocorrem. Assim sendo, tem-se como objetivo conhecer e descrever a percepção de qualidade de vida desses pacientes internados sob o olhar do próprio doente.

\section{MÉTODOS}

Estudo Transversal, de natureza observacional e exploratório-descritivo. Realizada em uma Instituição de longa permanência do interior paulista, durante o período de outubro a dezembro 2018. A população do estudo foi composta por pacientes internados na Instituição, maiores de 18 anos, portadores de doença crônica ameaçadora da vida, entre outras patologias severas. Para a coleta de dados utilizou-se um formulário elaborado pelo pesquisador contendo as características do paciente (dados sócio-demográficos como: idade, sexo, religião, estado civil, renda familiar, cor, escolaridade e presença de cuidador e o seu grau de parentesco) e dados clínicos (doença de base e comorbidades). Também foi aplicado a Palliative Scale Outcames Scale (POS), durante o período de internação do paciente.

A POS é instrumento específico para avaliação da qualidade de vida e resultados da saúde de doentes em cuidados paliativos. Desenvolvida originalmente por um grupo de pesquisadores do King's College de Londres, Hearn J e Higginson IJ 1999, a A POS é um instrumento específico para avaliação da qualidade de vida e resultados da saúde de doentes em cuidados paliativos. Desenvolvida originalmente por um grupo de pesquisadores do King's College de Londres, Hearn J e Higginson IJ 1999, a POS é uma escala QV utilizada em casos de doenças crônico-degenerativas em pacientes paliativos (WENTLANDT, et.al., 2012). É um instrumento de fácil domínio contendo perguntas nos diversos âmbitos, dentre eles espirituais, psico-sociais e físicos. As questões totalizam 40 pontos; 0 contempla a ausência de problemas e 40 uma pior qualidade de vida. Foi apresentada a estatística descritiva com as características sócio demográficas da amostra deste estudo. Os dados obtidos por meio da escala POS foram transportados para o programa SPSS para descrevêlos.

Conforme preconiza a Resolução no 466/2012 do Conselho Nacional de Saúde /Ministério da Saúde sobre diretrizes e normas regulamentadoras com pesquisa com seres humanos, antes de serem entrevistados os sujeitos da pesquisa foram esclarecidos quanto às entrevistas, sobre os objetivos da pesquisa, sobre 0 anonimato e sobre o direito de participarem ou não, tiveram todos manifestados sua aquiescência por meio da assinatura do termo de consentimento livre e esclarecido. Este protocolo de projeto de pesquisa foi encaminhado e aprovado sob o número 2.627.089 pelo Comitê de Ética em Pesquisa em Seres Humanos da Universidade de Marília - UNIMAR/ABHU.

\section{RESULTADOS}

O número de internados na referida instituição totaliza 53 pacientes. Destes, 30 pacientes não apresentavam condições cognitivas para responder o formulário, portanto 23 o fizeram. A partir dos dados colhidos no questionário sócio demográfico obtivemos a prevalência do sexo feminino, cor branca, estado civil viúvo, idade maiores de 70 anos, grau de instrução sem ciclo educacional completo, profissão trabalhador rural, religião católica, renda familiar de um salário mínimo, com presença de cuidador, portadores de doença de base (Tabela 1). 
Tabela 1 - Caracterização sócio demográfica da amostra. Marília, SP, 2018.

\begin{tabular}{|c|c|c|}
\hline Variável & $\mathbf{N}$ & Valor (\%) \\
\hline \multicolumn{3}{|l|}{ Sexo } \\
\hline Feminino & 12 & 52,2 \\
\hline Masculino & 11 & 47,8 \\
\hline \multicolumn{3}{|l|}{ Idade (anos) } \\
\hline 50 a 59 & 1 & 4 \\
\hline 60 a 69 & 6 & 26,1 \\
\hline 70 a 79 & 8 & 34,8 \\
\hline 80 ou mais & 8 & 34,8 \\
\hline \multicolumn{3}{|l|}{ Cor Referida } \\
\hline Branco & 17 & 73,91 \\
\hline Negro & 5 & 21,74 \\
\hline Pardo & 1 & 4 \\
\hline \multicolumn{3}{|l|}{ Renda Familiar } \\
\hline Até1 salário mínimo & 17 & 73,9 \\
\hline De 1 a 2 salários mínimos & 1 & 4,3 \\
\hline De 3 a 4 salários mínimos & 1 & 4,3 \\
\hline Mais de 5 salários mínimos & - & - \\
\hline Sem respostas & 4 & 17,4 \\
\hline \multicolumn{3}{|l|}{ Estado Civil } \\
\hline Solteiro(a) & 8 & 34,8 \\
\hline Casado(a) & - & - \\
\hline Divorciado(a) & 3 & 13 \\
\hline Viúvo(a) & 12 & 52,2 \\
\hline Vive Conjugalmente com alguém & - & - \\
\hline \multicolumn{3}{|l|}{ Nível de Escolaridade } \\
\hline Sem Ciclo Educacional Completo & 12 & 52,2 \\
\hline Ensino Fundamental Completo & 4 & 17,4 \\
\hline Ensino Médio & 4 & 17,4 \\
\hline Ensino Superior & 1 & 4,3 \\
\hline Outro (Não Estudou) & 2 & 8,7 \\
\hline \multicolumn{3}{|l|}{ Nível de Escolaridade } \\
\hline Sem Ciclo Educacional Completo & 12 & 52,2 \\
\hline Ensino Fundamental Completo & 4 & 17,4 \\
\hline Ensino Médio & 4 & 17,4 \\
\hline Ensino Superior & 1 & 4,3 \\
\hline Outro (Não Estudou) & 2 & 8,7 \\
\hline
\end{tabular}

Fonte: Zutin TLM et al, 2020. 
Tabela 2 - Resposta à questão quanto à presença de dor. Marília, SP, 2018.

\begin{tabular}{lcc}
\hline Opções & Total & Percentil \\
\hline $\begin{array}{l}\text { - Não } \\
\begin{array}{l}\text { 1. Ligeiramente - mas não o suficiente } \\
\text { para as esquecer }\end{array}\end{array}$ & 10 & $43 \%$ \\
$\begin{array}{l}\text { 2. Moderadamente - limitaram alguma da } \\
\text { minha atividade }\end{array}$ & 7 & $30 \%$ \\
$\begin{array}{l}\text { 3. Bastante - as minhas atividades ou a } \\
\text { concentração foram muito afetadas }\end{array}$ & 1 & $17 \%$ \\
$\begin{array}{l}\text { 4. Horrivelmente - incapaz de pensar em } \\
\text { qualquer outra coisa }\end{array}$ & 1 & $4 \%$ \\
\hline
\end{tabular}

Fonte: Zutin TLM et al, 2020.

Quando questionados sobre estar sentindo dores nos últimos 3 dias que afetam suas atividades diárias, $43 \%$ responderam que não são afetados. Outros 30\% referem que são ligeiramente prejudicados, $17 \%$ possuem ma moderada limitação nas atividades, $4 \%$ referiram atividades ou concentração muito afetadas e o restante de $4 \%$ relatou incapacidade de pensar em outra coisa (Tabela 2 ).

Ao serem indagados sobre ter sentido afetado por outros sintomas como enjoos, tosse ou prisão de ventre nos últimos 3 dias, $70 \%$ afirmaram não serem prejudicados por nenhum desses sintomas. Já $22 \%$ referem que são pouco afetados, $4 \%$ moderadamente afetados e outros $4 \%$ muito afetados.

Também obtivemos um percentil de $70 \%$ dos idosos entrevistados que não se sentem ansiosos ou preocupados com a sua doença crônica ou com o tratamento que é oferecido pela Instituição de longa permanência.

Tabela 3 - Resposta à questão quanto a preocupação e/ou ansiedade dos familiares e amigos com o doente. Marília, SP, 2018.

\begin{tabular}{lcc}
\hline Opções & Total & Percentil \\
\hline 0. Não, nada & 13 & $57 \%$ \\
1. De vez em quando & 7 & $30 \%$ \\
$\begin{array}{l}\text { 2. Às vezes - parece afetar a } \\
\text { concentração deles }\end{array}$ & 1 & $4 \%$ \\
3. A maior parte das vezes & 2 & $9 \%$ \\
4. Sim, sempre preocupados comigo & 0 & $0 \%$ \\
\hline
\end{tabular}

Fonte: Zutin TLM et al, 2020.

Houve a prevalência de $57 \%$ dos idosos entrevistados que seus familiares ou amigos não têm andado ansioso ou preocupados com o doente (Tabela 3). Assim como é possível afirmar que $65 \%$ dos entrevistados possuem toda informação necessária sobre sua condição e se sentem à vontade para perguntar o que desejam. Verificou-se que o estado deprimido não foi apontado por $35 \%$ dos idosos. Apenas $39 \%$ referem sentir se deprimido de vez em quando, e apenas $26 \%$ sentem-se deprimidos. Ademais, no que diz respeito à sensação de bem-estar consigo, nos últimos 3 dias, obtivemos resposta positiva por $87 \%$ dos idosos (Tabela 4). 
E então, os dados obtidos a respeito da resolução de problemas práticos, financeiros ou pessoais, resultantes da doença nos últimos três dias mostram que $91 \%$ tiveram problemas práticos que foram resolvidos e os assuntos estão em dia como desejariam ou não tiveram problemas práticos, $4 \%$ tiveram alguns problemas práticos que estão a ser resolvidos, enquanto $4 \%$ tiveram alguns problemas práticos que não foram resolvidos.

Tabela 4 - Resposta à questão sobre sentir-se deprimido. Marília, SP, 2018.

\begin{tabular}{lcc}
\hline Opções & Total & Percentil \\
\hline 0. Não, nunca & 8 & $35 \%$ \\
1. De vez em quando & 4 & $17 \%$ \\
2. Às vezes & 5 & $22 \%$ \\
3. A maior parte das vezes & 4 & $17 \%$ \\
4. Sim, sempre & 2 & $9 \%$ \\
\hline
\end{tabular}

Fonte: Zutin TLM et al, 2020.

\section{DISCUSSÃO}

Tendo por base a amostra composta por 23 participantes encontramos que a qualidade de vida está influenciada por diversos fatores, dentre os quais, se destacam dor, ansiedade, depressão, convívio social e econômico. A dor é considerada um dos grandes problemas de saúde pública atual segundo a Sociedade Brasileira para Estudo da Dor. Segundo Dellaroza MSG, et al. (2008), a dor crônica é definida como uma dor persistente por mais de seis meses e a dor aguda como um curto período - horas ou semanas. A dor que mais afeta a população mundial é a crônica, entre 20 e $40 \%$ já tiveram em algum momento da vida; acarretando prejuízos tanto no trabalho quanto no convívio social.

De acordo com estudo realizado por Waterkemper R e Reibnitz KS (2010), verificou-se que 70 a $90 \%$ dos pacientes em cuidados paliativos entrevistados afirmaram já ter sofrido com alguma dor, o que vem em contraposto com pacientes internados em uma Instituição de longa permanência com uma doença que ameaça a vida, pois $43 \%$ referiram não apresentar nenhuma dor nos últimos 3 dias.

Entretanto, ao serem indagados sobre algum outro sintoma (como tosse, enjoo ou prisão de ventre) $70 \%$ disse não apresentar. Assim, podemos pensar a respeito da qualidade de vida desses pacientes que mesmo com o seu envelhecimento e a presença das doenças crônicas ameaçadoras da vida, apresentam-se em uma ótima percepção. Porém, vale uma ressalva já mencionada, em que qualidade de vida está intimamente relacionado ao bem-estar físico, psicossocial, espiritual, econômico e cultural (CARVALHO MS, 2014).

Diante destes resultados obtidos é passível uma reflexão sobre qualidade de vida dessas pessoas. A qualidade de vida de qualquer indivíduo depende de fatores sociais, físicos, funcionais e psicológicos. O desequilíbrio entre esses coeficientes, como falta de contatos pessoais ou presença de patologias, causa vulnerabilidade e fragilidade do idoso, que refletindo diretamente na sua qualidade de vida. Ainda há aqueles que não se deixam afetar com a perda dos familiares, isolamento ou dificuldade em realizar as atividades de vida diárias, se adequando as circunstâncias (CARVALHO MS, 2014). Quando não são afetados pela sensação de impotência e possuem sua funcionalidade preservada, os idosos institucionalizados tendem a manter dentro da normalidade a questão da ansiedade.

Por seu caráter subjetivo, segundo Neri AL (2005), algumas observações devem ser destacadas quando se fala em qualidade de vida: ela não é uma responsabilidade individual e deve-se estender-se a toda população. A qualidade de vida assim, permeia construções sociais que tangem acesso a saúde e condições dignas de trabalho. O bem-estar de uma população não pode ser pensado singularmente, mas de modo 
ampliado a toda rede social do indivíduo. Seus fatores culturais, econômicos e sociais devem fazer parte dessa discussão para que ela seja efetivamente contemplada.

Uma pesquisa realizada por Gouveia PIA (2014), indicou que a maior parte dos entrevistados (68\%) possui preocupações e, destes, mostram predominância de preocupações de ordem física $(50 \%)$ e familiares $(31,8 \%)$. Aqueles que demonstraram preocupações físicas tendem a apresentar mais mal-estar emocional em relação aos outros (GOUVEIA PIA, 2014). De acordo com um estudo realizado para avaliar a prevalência da ansiedade em uma população de idosos institucionalizada em um município de Rondônia, concluiu-se que $92,0 \%$ da população estudada apresentava sintomas de ansiedade. Deste percentil, prevaleceu a ansiedade de grau leve. Azevedo JF (2009), já em outra pesquisa, verificou que a ansiedade coexiste em portadores de dor crônica (SANTOS KAS, et al., 2017).

Considerando a prevalência de idosos que não apresentam comorbidade $(73,91 \%)$ e mesmo aqueles que têm doença de base (como Hipertensão arterial crônica e Diabetes mellitus tipo 2), e pelos resultados obtidos pelo estudo de Santos KAS et al. (2017), é possível verificar a predominância de idosos que não se sentem ansiosos ou queixam de ansiedade justamente por não portarem dor crônica. Ademais, os motivos que a família têm para institucionalizar um idoso geralmente assumem uma conotação negativa (GOUVEIA PIA, 2014). A partir das limitações do idoso e a dependência que ele tem em relação à família, pode originar um estresse (CARVALHO MS, 2014). No contexto de uma doença crônica, diante de uma evolução lenta, são gerados desgastes nos laços familiares. $E$ ainda existem razões econômicas ou emocionais que levam a família a optar pela institucionalização do idoso (GOUVEIA PIA, 2014).

A literatura indica que após a institucionalização são poucas as famílias que retornam para visitá-los. Outro estudo feito, mostrou que apenas $10 \%$ dos idosos questionados recebem visitas diárias (GOUVEIA PIA, 2014). Isto pode refletir no resultado obtido, no qual a maioria dos idosos entrevistados referiu que a fam ília não se sente acometida. Quando foram questionados, muitos referiram não ter mais contato com o seio familiar e apontaram outros idosos da mesma instituição como amigo.

De acordo com o exposto, também é possível entender o motivo da solidão que acomete maior parte dos idosos institucionalizados. Um estudo abordou esta questão; Barroso VL (2006), comparando a presença da solidão entre esses e idosos não institucionalizados, concluiu que existe relação com o local em que vivem. Assim, aqueles que se encontram institucionalizados possuem maior incidência de sentimentos de isolamento. Em contrapartida, nesse mesmo estudo, idosos institucionalizados que ainda estão inseridos no contexto familiar demonstraram menores índices de sentimentos de solidão. Além disso, a proximidade desses idosos com amigos, havendo a integração do idoso em todo um contexto social, está relacionada com menores sentimentos de depressão e ansiedade (BARROSO VL, 2006).

A comunicação com o paciente e informações sobre seu estado de saúde são de extrema importância para manter um laço de confiança com o doente e sua família. Essas informações devem ser dadas de forma simples e gradual, sempre respeitando a quantidade de informação que o paciente suporta receber (GOMES ALZ e OTHERO MB, 2016).

Comunicar-se e saber se comunicar com o paciente é fundamental para que os doentes se sintam mais à vontade e mais confiantes em relação ao médico (GOULART BNG e CHIARI BM, 2010). Quando a comunicação entre médico e paciente não acontece, pode levar a três situações: o médico pode não compreender as palavras usadas pelos pacientes, o paciente pode não entender os termos utilizados pelo médico e/ou o paciente pode ter dificuldade em aderir ao tratamento (GOULART BNG e CHIARI BM, 2010). Desse modo, o paciente se sentirá mais confortável diante de informações sobre seu estado, sabendo lidar melhor com sua condição física e mental.

Observou-se que a dependência física trazida pela idade avançada somada a instabilidade econômica aumentam a procura pela institucionalização de idosos, o que acarreta em adaptação a novas rotinas e ao distanciamento da família (PORCU M, et al., 2002). Do ponto de vista epidemiológico, observa Coutinho MPL et al. (2002) que os sintomas depressivos vêm aumentando na população em geral, mas especialmente entre idosos e ainda mais elevados em algumas populações, como hospitalizados ou institucionalizados, porém 
neste estudo foi observado que os pacientes institucionalizados não apresentavam este quadro depressivo, contrapondo o estudo (COUTINHO MPL, et al., 2002).

Idosos com melhores relações afetivas entre seus familiares e amigos lidam mais facilmente com suas condições de envelhecimento, estabelecendo menores índices de solidão e depressão, uma vez que a perda de pessoas conhecidas gera um sentimento de que sua morte se aproxima (BARROSO VL, 2006). Segundo um estudo realizado por Oliveira M, et al. (2016), é notável a piora na condição de saúde em pacientes que estão próximos da morte, portanto, passou a ser importante priorizar sua qualidade de vida, visando minimizar as consequências de doenças crônicas progressivas.

Com o envelhecimento da população já consolidado, apontado por Oliveira M, et al. (2016), observamos a crescente necessidade de adequação dos modelos de atenção à saúde para a população idosa, com enfoque na promoção da saúde, ações educativas, cuidado precoce e reabilitação, como linha de cuidado ideal, aliada a incorporação deles à sociedade e ao convívio familiar, como meios para se atender de forma justa essa parcela populacional (LIMA-COSTA MF e VERAS RP, 2003). Portanto, o presente estudo demonstra que as necessidades da nossa população amostral têm sido satisfeitas, fazendo-nos concluir que provavelmente a expectativa da qualidade de vida nesses pacientes possa estar aquém da ideal por conta de seu contexto clínico e social, e não pela excelência do suporte familiar e social, considerando as dificuldades encontradas. Já que encontramos um percentil de $87 \%$ de satisfação pessoal.

Segundo Deponti RN e Acosta MAF (2010) o envelhecimento saudável consiste em uma visão multidimensional, sendo englobados fatores biológicos, físicos e sociais. Dessa forma, observa-se a necessidade de um equilíbrio entre diversas dimensões. Por isso os dados obtidos em que houve prevalência da resolução de problemas práticos, financeiros ou pessoais refletem em maior probabilidade de equilíbrio nas diversas áreas que compõem a qualidade de vida.

De acordo com a OMS envelhecimento ativo é "o processo de otimização das oportunidades de saúde, participação e segurança, com o objetivo de melhorar a qualidade de vida à medida que as pessoas ficam mais velhas". Ou seja, além da ausência do processo saúde-doença é necessário o bem-estar mental e social, prezando para o estabelecimento de autonomia e independência dos idosos. Permitindo que eles possuam direitos para atuar na vida em comunidade. A partir do momento em que as políticas sociais de saúde estiverem implantadas haverá intensa melhora na qualidade de vida dos idosos, redução de deficiências associadas a doenças crônicas da terceira idade e redução de gastos de assistência médica, para isso são necessárias as políticas sociais de saúde, mercado de trabalho, emprego e educação apoiem o envelhecimento ativo.

Alguns fundamentos da Política Nacional de Saúde da Pessoa Idosa atuam de forma que, se realizados com sucesso, promoveriam o envelhecimento ativo, já que eles são participação ativa dos idosos na sociedade, no desenvolvimento e na luta contra a pobreza; fomento à saúde e bem-estar na velhice promovendo o envelhecimento saudável; criação de um ambiente propício e favorável ao envelhecimento; além de fomento a recursos sócios educativos e de saúdes direcionadas ao atendimento ao idoso.

Dessa maneira, com o consequente envelhecimento ativo, também conhecido como envelhecimento saudável, a capacidade cognitiva da terceira idade será melhor desenvolvida, já que ela é diretamente relacionada com todos os fatores anteriormente citados, e, portanto, haverá maior facilidade em resolver problemas práticos.

\section{CONCLUSÃO}

Obtivemos a prevalência do sexo feminino, cor branca, viúvo, maiores de 70 anos, sem ciclo educacional completo, trabalhador rural, religião católica, renda familiar de um salário mínimo, com presença de cuidador e portadores de doença de base. Essa maioria referiu não sentir dores que afetem suas atividades diárias. Afirmaram ainda, possuir todo respaldo necessário sobre sua condição e referiram receber a ajuda quando necessário. Dessa forma, concluímos que a qualidade de vida desses pacientes em Instituições de longa permanência é satisfatória às necessidades físicas, sociais e psicológicas. 


\section{AGRADECIMENTOS}

Agradecemos a Universidade de Marília que nos apoiou nesta pesquisa, principalmente os nossos professores que sempre nos estimularam.

\section{REFERÊNCIAS}

1. ARAÚJO MMT, SILVA MJP. A comunicação com o paciente em cuidados paliativos: valorizando a alegria e o otimismo. Revista da Escola de Enfermagem da USP, 2007; 41(4): 668-674.

2. 2.AZEVEDO JF. Prevalência de depressão e ansiedade em idosos institucionalizados no município de Ji-Paraná Rondônia. Dissertação (Programa de Pôs-Graduação em Ciências da Saúde) - Faculdade de Ciências da Saúde, Universidade de Brasília, Brasília. 2009, 79p.

3. BARROSO VL. Órfãos geriatras: sentimentos de solidão e depressividade face ao envelhecimento - estudo comparativo entre idosos institucionalizados e não institucionalizados. Monografia (Licenciada em Psicologia pelo Instituto Superior de Psicologia Aplicada) - Instituto Superior de Psicologia Aplicada - ISPA, Portugal, 2006, 13p.

4. BYOCK I. Dying well: The prospect for growth at the end of life. ThorndikePr, 1997; 583p.

5. CALDAS CP. O idoso em processo de demência: o impacto na família. In: MINAYO, MCS., and COIMBRA JUNIOR, CEA., orgs. Antropologia, saúde e envelhecimento [online]. Rio de Janeiro: Editora FIOCRUZ, 2002. Antropologia \& Saúde collection, pp. 51-71.

6. CARVALHO MS. O Cuidado Paliativo a idosos institucionalizados: vivência dos cuidadores. Dissertação (Mestrado em Cuidados Paliativos) - Faculdade de Medicina da Universidade do Porto, Portugal, 2014; 205p.

7. COUTINHO MPL, et al. Depressão, um sofrimento sem fronteira: representações sociais entre crianças e idosos. Psico-USF, 2002;8(2):183-192.

8. DALLA VECCHIA R, et al. Qualidade de vida na terceira idade: um conceito subjetivo. Rev. bras. Epidemiol., 2005; 8(3): 246-52.

9. DELLAROZA MSG, et al. Caracterização da dor crônica e métodos analgésicos utilizados por idosos da comunidade. Rev. Assoc. Med. Bras, 2008;54(1):36-41

10. 10.DEPONTI RN, ACOSTA MAF. Compreensão dos idosos sobre os fatores que influenciam no envelhecimento saudável. EstudlnterdiscipEnvelhec.,2010;15(1):33-52.

11. GOMES ALZ, OTHERO MB. Cuidados Paliativos. Estudos Avançados, 2016;30(88):155-166.

12. GOULART BNG, CHIARI BM. Humanização das práticas do profissional de saúde: contribuições para reflexão. Ciência \& Saúde Coletiva, 2010;15(1): 255-268.

13. GOUVEIA PIA. Avaliação do sofrimento emocional em idosos institucionalizados em lares. Dissertação (Mestrado em Cuidados Paliativos) - Faculdade de Medicina da Universidade do Porto, Portugal, 2014, 116p.

14. HEARN J, HIGGINSON IJ. Development and validation of a core outcome measure for palliative care: the palliative care outcome scale. Palliative Care Core Audit Project Advisory Group. Qual Health Care, 1999 8(4): $219-27$.

15. LIMA-COSTA MF, VERAS RP. Saúde Pública e envelhecimento. CadSaúdePública, 2003;19(3):700-701.

16. NÈRI AL. As políticas de atendimento aos direitos da pessoa idosa expressa no Estatuto do Idoso. A Terceira Idade,2005;16(34):7-24.

17. OLIVEIRA M, et al. Idoso na saúde suplementar: uma urgência para a saúde da sociedade e para a sustentabilidade do setor. Rio de Janeiro: Agência Nacional de Saúde Suplementar, 2016; 134p.

18. PORCU M, et al. Estudo comparativo sobre a prevalência de sintomas depressivos em idosos hospitalizados, institucionalizados e residentes na comunidade. Acta Sci, 2002;24(3):713-717.

19. SANTOS KAS, et al. Transtorno de ansiedade em idosos com dor crônica: frequência e associações. Rev. Bras. Geriatr. Gerontol., 2017;20(1):95-102.

20. SILVA MMP. Qualidade de vida em cuidados paliativos: análise comparativa da percepção dos doentes e profissionais. Dissertação (Mestradoem Gestão e Economia da Saúde) - Faculdade de Economia da Universidade deCoimbra, Portugal, 2014; 102p.

21. WATERKEMPER R, REIBNITZ KS. Cuidados paliativos: a avaliação da dor na percepção de enfermeiras RevGaúchaEnferm., 2010:31(1); 84-91.

22. WENTLANDT K, et al. Referral practices of oncologists to specialized palliative care. Journal of Clinical Oncology, $2012 ; 30(35): 4380-6$. 